\title{
A potential molecular mechanism for regulating pre-mRNA splicing of implantation-related genes through unique uterine expression of splicing factor SC35 in women and rhesus monkeys
}

\author{
G-Y. Nie' , A. L. Hampton' ${ }^{1}$, G-Q. Fu¹,2, Y-X. Liu², J. K. Findlay ${ }^{1}$ \\ and L. A. Salamonsen ${ }^{1}$ \\ ${ }^{1}$ Prince Henry's Institute of Medical Research, PO Box 5152, Clayton, Victoria 3168, \\ Australia; and ${ }^{2}$ State Key Laboratory for Reproductive Biology, Institute of Zoology, \\ Chinese Academy of Sciences, Beijing 100080, China
}

\begin{abstract}
Splicing factor SC35 is an essential component of the spliceosome, the cellular apparatus that removes introns from pre-mRNA to provide alternatively spliced isoforms. Many proteins associated with development of uterine receptivity and embryo implantation are present as isoforms, the tissue-specific expression of which may be regulated through alternative splicing. SC35 was identified as being increased at implantation sites during early pregnancy in mice. However, the present study has demonstrated that SC35 is present in human and rhesus monkey endometrium, that the protein is increased during the secretory phase of the oestrous cycle compared with the proliferative phase in both these primates and that it is present in a distinct pattern within the nucleus of both epithelial and stromal cells, as well as in cells of the
\end{abstract}

\section{Introduction}

Embryo implantation is a key factor in the establishment of pregnancy. For implantation to be achieved successfully, the endometrium must acquire a state of receptivity. The endometrium undergoes considerable morphological and molecular changes, which are driven by the ovarian steroid hormones, oestrogen and progesterone. A number of genes and their protein products are regulated in a manner appropriate for a role in implantation. Several of these molecules have been shown in functional studies to be critically important for this process in rodents. Although it is not possible to perform studies on early implantation in women, a number of these molecules have been shown by correlative studies to be of potential importance for implantation in humans and primates (for example leukaemia inhibitory factor (LIF): Hambartsoumian, 1998; Vogiagis and Salamonsen, 1999; interleukin II (IL-II): Dimitriadis et al., 2000; calcitonin: Kumar et al., 1998).

On the basis of the hypothesis that there would be a number of previously unidentified molecules that are

Email: guiying.nie@med.monash.edu.au vasculature. Both the intensity of immunoreactive protein and the proportion of cells that stain for SC35 alter with the phase of the oestrous cycle. A very precise expression pattern of SC35 (both protein and mRNA) was seen during early placentation in rhesus monkeys. At implantation sites between day 24 and day 35 of early pregnancy, SC35 was expressed strongly in cytotrophoblasts within the trophoblastic shell, in syncytiotrophoblast at the periphery of the cell column and in both cytotrophoblast and syncytiotrophoblast in the floating villi. In the adjacent maternal decidua, expression of SC35 was weak. These results indicate a role for SC35 in preparation of a receptive uterus, in the provision of secreted proteins to support blastocyst development and in trophoblast invasion.

critical for implantation, the technique of RNA differential display PCR was used (Liang and Pardee, 1992, 1993) and the mRNA expression pattern of implantation and interimplantation sites in the mouse uterus was compared on day 4.5 of pregnancy (day $0=$ day of vaginal plug), just when attachment of the embryo to the endometrial epithelium is commencing (Nie et al., 2000a,b). One of the genes identified as being differentially regulated between the two sites was splicing factor SC35, an essential component of the spliceosome (Nie et al., 2000c). SC35 protein is required for the first step in the splicing of precursor mRNA (pre-mRNA) (Fu and Maniatis, 1992). In mice, the overall expression of SC35 mRNA was much higher in implantation sites than in inter-implantation sites in the uterus during early pregnancy. Expression of SC35 mRNA was increased as early as day 3.5, but increased further only at implantation sites on day 4.5 and remained high up to day 8.5. On day 10.5, SC35 mRNA expression was increased in the embryonic portion of implantation sites. During the normal oestrous cycle, expression was low at pro-oestrus and oestrus, increased during metoestrus and started to decline during dioestrus. Measurement of SC35 mRNA in ovariectomized mice treated with steroid hormones indicated that progesterone was able to 
upregulate uterine SC35 in mice, whereas examination of uteri of pseudo-pregnant mice supported a regulatory role also for the embryo itself (Nie et al., 2000c).

SC35 is a member of the serine-argenic (SR) protein-like factors protein family and an essential component of the spliceosome, the cellular apparatus that removes introns from pre-mRNA. Many of the proteins associated with development of uterine receptivity and embryo implantation occur as alternatively spliced isoforms, the tissue-specific expression of which may be regulated by pre-mRNA processing. Correct processing of the pre-mRNAs is thus clearly important for the accurate expression of these vital proteins during early pregnancy and it follows that appropriate expression of SC35 splicing factor is likely to be pivotal at implantation. In particular, because of the concentration-dependent action of SC35 splicing factor in the splicing event (Fu et al., 1992), higher expression of this splicing factor at implantation sites rather than at inter-implantation sites may influence the pattern of splicing of genes at these sites and the accurate expression of alternative protein products.

Although there are many similarities between the events at implantation in mice and women, there are also considerable differences. Specifically, preparation of the endometrium for implantation during each menstrual cycle in women is much more extensive, even in the absence of an embryo. The decidual transformation of stromal fibroblasts, which is induced only by a stimulus such as an implanting blastocyst in mice, occurs spontaneously during the late secretory phase in women. In addition, although the expression of a number of genes that are important for development of uterine receptivity in mice is very tightly regulated both temporally and spatially, human homologues of such genes are often expressed throughout the cycle but with an increase in the mid-secretory phase that is maintained until menstruation (Vogiagis and Salamonsen, 1999; Dimitriadis et al., 2000). Thus, it is appropriate to establish the pattern of expression during the normal menstrual cycle to identify whether a specific gene product might be critical for implantation in women.

It was hypothesized that SC35 protein would be important for the establishment of early pregnancy in women and, thus, the temporal and spatial location of this protein was examined in human endometrium, sampled across the normal menstrual cycle and in the endometrium of the rhesus monkey, both during the oestrous cycle and at implantation sites during normal early pregnancy. The immunohistochemical data were verified by both western blot analysis and in situ hybridization. The pattern of expression of this protein indicates that it is likely to play a role in processing specific pre-mRNAs during the establishment of pregnancy in primates.

\section{Materials and Methods}

\section{Tissue collection and processing}

Endometrial tissue was obtained at curettage from women with regular menstrual cycles and no apparent endometrial dysfunction, who gave informed consent for collection of tissue. Approval was given by the Human Ethics Committee at Monash Medical Centre, Melbourne. The women were either of proven fertility and scheduled for tubal ligation or were undergoing tests for patency of the Fallopian tubes. Patients with leiomyomas, endometriosis or those whom had received any steroid treatment during the past 12 months were specifically excluded from the study. Tissue samples were fixed in buffered formalin at $4{ }^{\circ} \mathrm{C}$ overnight, washed in Tris-buffered saline (TBS) ( $\mathrm{pH} 7.4)$ and processed to paraffin wax blocks. Tissue was dated initially from the patient's testimony and confirmed histologically by a qualified gynaecological pathologist. For the immunohistochemical study, the numbers of tissues examined in each phase were: nine menstrual, ten proliferative, five preovulatory (days 13-14), nine early secretory, ten midsecretory and nine late secretory.

Rhesus monkeys (Macaca mulatta) were maintained in the Fu-Zhou Primate Research Centre, China. All experimental work was approved by the Animal Ethics Committee at the Institute of Zoology, Chinese Academy of Sciences. Menstrual cycles of female monkeys were monitored for two to three cycles before sampling. Uterine tissue was collected 1 day before (ov-1, $n=3)$, and at day $5(\mathrm{ov}+5$; $n=3)$, day $10(\mathrm{ov}+10 ; n=3)$ and day $15(\mathrm{ov}+15 ; n=2)$ after ovulation. For pregnant monkeys, animals were permitted to mate over a period of 3 days at the anticipated time of ovulation. The second day of mating was designated day 0 of pregnancy. The presence of a conceptus was confirmed by ultrasound diagnosis and rectal examination. At the appropriate time of either the oestrous cycle or pregnancy, the monkeys were killed humanely, the uterus (with or without placenta) was removed, and appropriately selected wedges of full thickness uteri (myometriumendometrium-placenta of $<0.5 \mathrm{~mm}$ in diameter) were fixed in buffered formalin overnight at $4^{\circ} \mathrm{C}$, washed in TBS $(\mathrm{pH}$ 7.4) and processed to paraffin wax blocks. Samples of uterus, including placenta when available, were collected from pregnant monkeys between day 16 and day $35(n=17)$ of pregnancy: some samples were from implantation sites. When appropriate, tissue was also taken from the secondary placenta $(n=3)$.

\section{Immunohistochemistry}

Paraffin wax was removed from sections $(5 \mu \mathrm{m})$ of human endometrium or rhesus monkey uterus. The sections were then hydrated and subjected to immunohistochemistry for splicing factor (SC35) with a mouse anti-SC35 monoclonal antibody (clone no. SC-35; Sigma Chemical Co., St Louis, MO). Quality control was provided by including one slide from a single endometrial tissue block or single uterine block, respectively, in each staining run. As a negative control, a second section of each tissue was incubated with an irrelevant murine monoclonal antibody diluted to the same final protein concentration as the SC35 antibody. SC35 expression was investigated for a total of 52 
human tissues across the menstrual cycle, with between five and ten samples for each phase of the cycle: menstrual (days 1-4), proliferative (days 5-12), peri-ovulatory (days 13-15), early- (days 16-19), mid- (days 20-24) or late- (days 25-28) secretory phases. For the cyclic monkey tissues, three animals were used for each of ov $-1, \mathrm{ov}+5$ and ov +10 , and two animals were used for ov+15. Sections from 17 pregnant monkeys between day 16 and day 35 of pregnancy were also stained for SC35 expression.

All steps of the staining protocol for SC35 were performed at room temperature, except for the incubation with primary antibody, which was performed at $4^{\circ} \mathrm{C}$. Tissues were washed with TBS $(\mathrm{pH} 7.6)$ for $10 \mathrm{~min}$ and then incubated in $3 \%(\mathrm{v} / \mathrm{v})$ hydrogen peroxide for $5 \mathrm{~min}$, followed by further washing in TBS. After blocking of nonspecific binding with $10 \%$ normal horse serum in TBS for $30 \mathrm{~min}$, tissue sections were incubated overnight (15-18 h) in either anti-SC35 monoclonal antibody (1:500) or control antibody diluted appropriately in TBS. After washing, tissue sections were incubated in biotinylated horse anti-mouse IgG (Vector Laboratories Inc., Burlingame, CA) for 30 min. The Dako (Glostrup) strept-ABC-horseradish peroxidase system was used to detect cells expressing SC35, and colour was developed using the liquid DAB-plus substrate kit (Zymed, San Francisco, CA). Sections were counterstained with Harris' haematoxylin (1:10), and were then dehydrated and mounted. Microscopy was performed using an Olympus BH50 microscope fitted with a digital camera (Videocam Fujix HC-2000; Fuji Photo Film Co., Tokyo) coupled to a computer using Analytical Imaging Station (Imaging Research Inc.) and Adobe Photoshop software. For the tissues from cyclic women and monkeys, both the intensity of immunostaining and the percentage of cells stained in individual cellular compartments within each section were scored blind by two independent observers. In the rare instances in which scores were different, a mean was taken. For intensity of stain, zero represented no stain and three represented maximum staining in relation to the positive and negative controls. For the percentage of cells stained, a score of $1=0-30 \%, 2=30-70 \%$ and $3=70-100 \%$ of cells stained was recorded within any cellular compartment for the human tissues. For the monkey tissues, because of the greater variability, scores were assigned as: $1=1-10 \%$, $2=10-40 \%, 3=40-80 \%$ and $4=80-100 \%$ of cells stained within the particular cellular compartment. Results are presented as mean \pm SEM. For the tissues from pregnant monkeys, because of the variability of the quality of the implantation sites, descriptive analysis only was performed.

\section{In situ hybridization}

The immunohistochemical data were validated and which types of cell in the uterus express mRNA for SC35 were established by performing in situ hybridization on monkey implantation sites using sense and antisense digoxigenin (DIG)-labelled RNA probes generated against nucleotides 242-594 of the human SC35 mRNA sequence
(Accession no: M90104) using the DIG RNA labelling kit (Boehringer Mannheim, Nunawading, Victoria). The concentrations were determined according to the manufacturer's instructions. Five micron sections of formalinfixed paraffin wax-embedded monkey tissues were subjected to in situ hybridization. The sections were postfixed with $4 \%(\mathrm{v} / \mathrm{v})$ paraformaldehyde for $20 \mathrm{~min}$, washed with TBS and digested with proteinase $\mathrm{K}\left(25 \mathrm{ng} \mathrm{ml}^{-1}\right.$ in TBS) for $20 \mathrm{~min}$ at $37^{\circ} \mathrm{C}$. The tissue sections were then dehydrated in a graded series (increasing concentrations) of ethanol, followed by overnight hybridization at $42^{\circ} \mathrm{C}$ with 10-20 ng DIG-labelled RNA probe in $50 \mu$ l hybridization buffer consisting of $2 \times$ SSC $(1 \times$ SSC: $150 \mathrm{mmol} \mathrm{NaCl} \mathrm{I-1,}$ 15 mmol Tri-sodium citrate $\mathrm{I}^{-1}$, $\left.\mathrm{pH} 7.0\right), 50.0 \% \quad(\mathrm{v} / \mathrm{v})$ formamide, $10.0 \%(\mathrm{w} / \mathrm{v})$ dextran sulphate, $0.02 \%(\mathrm{w} / \mathrm{v}) \mathrm{SDS}$ and $0.1 \mathrm{mg}$ salmon sperm DNA $\mathrm{ml}^{-1}$. The sections were then washed twice for $30 \mathrm{~min}$ with $0.1 \times \mathrm{SSC}$ at $42^{\circ} \mathrm{C}$. After washing with buffer $\left(0.1 \mathrm{~mol}\right.$ Tris $\mathrm{I}^{-1}, 150 \mathrm{mmol} \mathrm{NaCl} \mathrm{I}^{-1}$, $\mathrm{pH}$ 7.5) for $10 \mathrm{~min}$ at room temperature, blocking solution containing $0.1 \%(\mathrm{v} / \mathrm{v})$ Triton-X100 and 15\% normal sheep serum in buffer was applied for $1 \mathrm{~h}$. Anti-DIG-horseradish peroxidase in blocking solution was then applied, after which the colour reaction was developed using diaminobenzidine (Zymed) for $3 \mathrm{~min}$ at room temperature. Sections were counterstained with Harris' haematoxylin. Sections hybridized with the corresponding sense probes served as negative controls. Hybridization specificity was verified by abolishment of signal after pre-treatment with RNase before hybridization with antisense riboprobe.

\section{Western blot analysis of human endometrium}

Proteins were extracted from two samples each of proliferative and secretory phase human endometrium. Tissue was then weighed and homogenized in $6.0 \%(\mathrm{w} / \mathrm{v})$

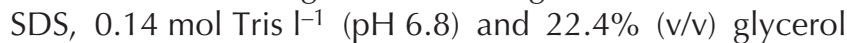
( $2 \mathrm{ml}$ per $100 \mathrm{mg}$ of tissue) with a protease inhibitor cocktail (Calbiochem, Croyden; $5 \mu \mathrm{l}$ per $100 \mathrm{mg}$ of tissue). The homogenate was then passed sequentially through 21, 23 and 25 gauge needles followed by centrifugation at $14000 \mathrm{~g}$ for $15 \mathrm{~min}$ at $4^{\circ} \mathrm{C}$. Fifteen micrograms of total protein from each supernatant, together with molecular weight markers (Kaleidoscope prestained standards; Biorad, Regent's Park, NSW) were subjected to SDS-PAGE on a $10 \%$ gel under reducing conditions. The proteins were transferred to Hybond-PTM membranes (Amersham Life Sciences, Sydney). After blocking non-specific binding sites with $5 \%(\mathrm{w} / \mathrm{v})$ skimmed milk powder in TBS with $0.1 \%(\mathrm{v} / \mathrm{v})$ Tween 20 for $1 \mathrm{~h}$, blots were incubated for $1 \mathrm{~h}$ at room temperature with a 1:1000 dilution of the SC35 antibody diluted in $5 \%(\mathrm{w} / \mathrm{v})$ skimmed milk powder in TBS with $0.1 \%$ $(\mathrm{v} / \mathrm{v})$ Tween 20. Western blots were then incubated with horseradish peroxidase-labelled sheep anti-mouse IgG (Amersham) and developed by chemiluminescence (ECL Plus system; Amersham). As a negative control, mouse IgG (Dako) at a similar concentration was used in place of primary antibody. 

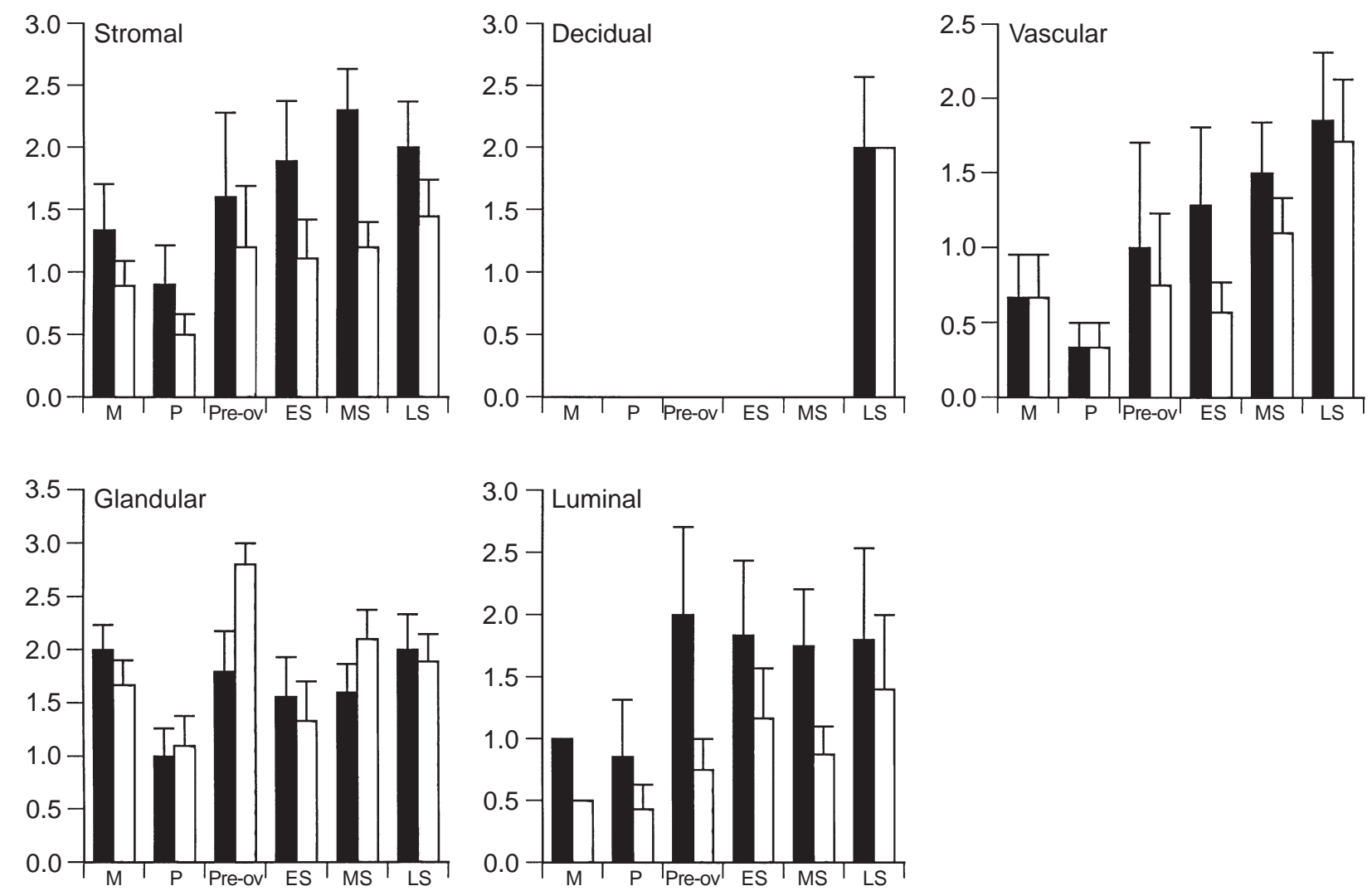

Fig. 1. Immunostaining of the splicing factor SC35 in human endometrium throughout the menstrual cycle. Mean intensity of immunostaining for SC35 ( $\square$ ) and the percentage of cells stained within a cellular compartment $(\square)$ in human endometrium during different phases of the menstrual cycle. Cellular compartments are stromal, decidual, vascular, glandular epithelium and luminal epithelium. The intensity of stain is represented on a scale of 0 (absent) to 3 (maximum staining) for each cellular compartment. The proportion of cells stained is also represented on a scale of $1-3$, where $1=1-30 \%, 2=30-70 \%$ and $3=70-100 \%$ of cells within a cellular compartment. Data are expressed as mean \pm SEM. Only two menstrual tissues had intact luminal epithelium so error bars are not provided. For other phases of the oestrous cycle, between five and ten tissues were scored. ES: early secretory; LS: late secretory; M: menstrual; MS: mid-secretory; P: proliferative; Pre-ov: preovulatory.

\section{Results}

\section{Localization of SC35 protein in human endometrium throughout the menstrual cycle}

A total of 52 samples was examined as detailed above. However, luminal epithelium and decidualized stromal cells were not present within every sample within a given phase. In particular, luminal epithelium was present only in two of nine menstrual samples and decidua only in three late secretory samples. SC35 was found in the nuclei of cells in all cellular compartments of the human endometrium: luminal and glandular epithelium, stroma, vasculature and in the decidualized stromal cells late in the menstrual cycle. In each cellular compartment, only a proportion of the cells was stained and staining varied in intensity with the phase of the menstrual cycle (Figs 1 and 2a-f). Under high power, it was clear that the antibody detected protein in discrete particles (Fig. 2e), a characteristic feature described for SC35 by Fu and Maniatis (1990).

Fig. 2. Photomicrographs of representative examples of immunostaining and in situ hybridization for SC35 protein and mRNA, respectively. (a-f) Immunostaining of human endometrium showing: (a) glands ( $\mathrm{gl}$ ) and stroma (s) in the proliferative phase and (b) in the mid-secretory phase, and (c) luminal epithelium and decidual cells (dc) in the late secretory phase and (d) spiral arterioles in the late secretory phase. pe: pericyte. (e) High power view of glands on day 28 showing speckled appearance (spec) of nuclear staining and (f) the negative control. (g-m) Immunostaining of rhesus monkey endometrium at (g) day 5 and (h) day 10 after ovulation. (i-m) An implantation site in the rhesus monkey at day 27 after ovulation. (i) A low power view of the implantation site, which is enlarged showing (j) trophoblast shell (ts), (k) floating villus (v) and (I) decidua, and (m) the negative control. ( $\mathrm{n}-\mathrm{q})$ In situ hybridization for SC35 mRNA in the same tissue as shown in (i). (n) Trophoblast shell, (o) floating villus and (p) decidua, and (q) the negative control. Scale bars represent $50 \mu \mathrm{m}$. 

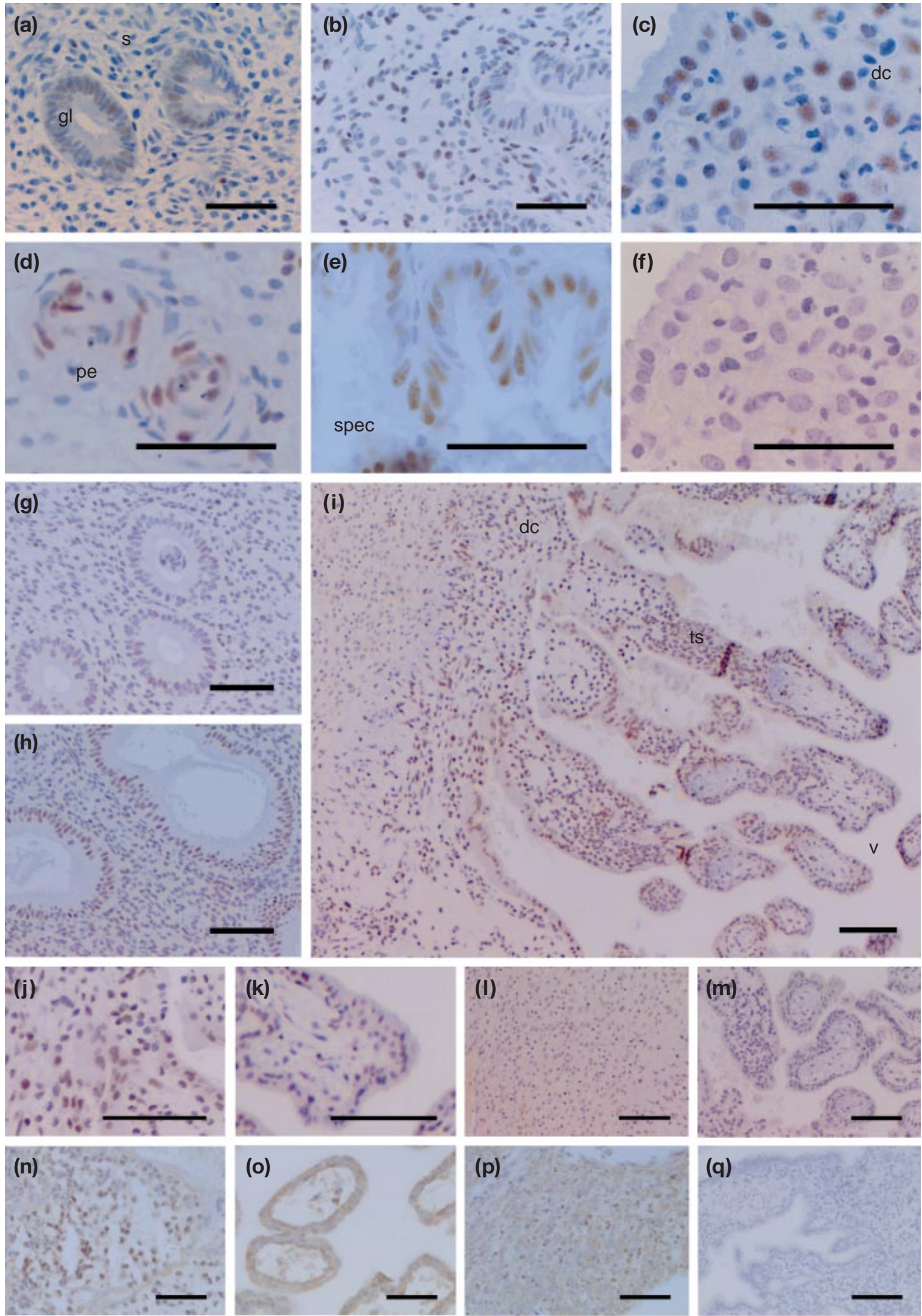

(q)

Fig. 2. For legend see facing page. 
Epithelium. In both luminal and glandular epithelium, the total number of cells stained was higher in the secretory than in the proliferative phases (Figs 1 and 2a,c). Overall, a lower proportion of the total number of cells was stained in luminal than in glandular epithelium. In the glands, there was a sharp increase in the number of positively stained cells during the periovulatory phase, whereas in the luminal epithelium it was the intensity of stain in positively stained cells rather than the proportion of cells stained that increased at this time. This intensity remained high throughout the secretory phase.

Stroma. During the proliferative phase of the menstrual cycle, only a very small percentage of stromal cells stained positive for SC35 and the staining was of low intensity (Figs 1 and 2a). During the preovulatory phase, this staining increased markedly and although the number of cells that stained remained constant throughout the secretory phase, the intensity of stain continued to increase until the midsecretory phase (Figs 1 and 2b). Importantly, after decidual transformation, most of the decidualized cells stained strongly for SC35 (Figs 1 and 2c).

Vasculature. As in the other compartments, both the percentage of cells stained and the intensity of stain in the pericytes increased with the progression of the oestrous cycle, and a substantial increase in the periovulatory phase was sustained and increased somewhat during the secretory phase (Figs 1 and 2d).

\section{Localization of SC35 protein in rhesus monkey endometrium across the menstrual cycle}

As for human endometrium, staining for SC35 was observed in all cellular compartments (Figs 2g, h and 3). As endometrium of full thickness was available from these animals (compared with only the functionalis layer in humans), and as differences were observed, glandular epithelium from both functionalis and basalis layers was scored separately. Some staining was observed in the myometrium but this was not analysed in detail as it is beyond the scope of the present study. Overall, the results from the present study show that SC35 protein in rhesus monkey endometrium was present in low amounts at ov-1 (Fig. 2g), increasing to a maximum at ov+10 (Fig. 2h), but then decreasing again at ov +15 (Fig. 3). Both the intensity of stain and the proportion of cells stained were higher in the outer glandular epithelium than in the luminal epithelium (Fig. 3), and the proportion of glands stained was slightly higher in the deep glands than in the outer glands. At ov +5 and ov+10, SC35 was detected in most (>75\%) of the glandular epithelial cells. Within the stroma, the staining was overall less intense than in the glands and again not all cells were stained. Maximum intensity of staining was seen in the stromal cells at ov +5 and ov +10 , but only $40-60 \%$ of the cells were positively stained even at this time (Fig. 3).
Localization of SC35 protein in rhesus monkey endometrium at implantation sites during early pregnancy

Within the uterus. In the uteri of pregnant rhesus monkeys, intense staining for SC35 was generally observed in the deep glands close to the myometrium. The glands closer to the uterine lumen stained less strongly. Where luminal epithelium was retained, only some cells (approximately 10\%) stained for SC35, although the intensity of staining was moderately strong. Staining was also observed in the endometrial stroma, although the intensity was quite variable between animals. When decidualized stroma was present, staining was present but was weak. Positive staining, sometimes quite strong, was also observed in perivascular cells.

At the implantation site. The earliest implantation site sampled was on day 24 and the latest on day 35 of pregnancy. Overall, the staining for SC35 was apparent in the same cellular compartments at all implantation sites between these days (Fig. 2i-l). The strongest staining was present in the cytotrophoblast cells in the trophoblast shell (Fig. 2j) and in the syncytial trophoblast on the exterior of the cell column. Less intense staining was observed in both cytotrophoblast and syncytiotrophoblast in the floating villi; there was no apparent difference in the expression between these cells (Fig. 2k). Nearly all cells of these types stained for SC35. Trophoblast cells were positively identified by immunostaining serial sections with a pan-cytokeratin antibody (C11; Santa Cruz Biotechnology, CA; data not shown). Staining in the trophoblast cells was stronger than that in the adjacent decidua (Fig. 2l), which was positively identified by its strong staining for prolactin in adjacent sections (data not shown). When tissue from the secondary placenta was available (three animals), neither the staining pattern nor the intensity of stain was different from that in the main placenta.

\section{Localization of SC35 mRNA}

Given that SC35 is an intracellular protein, in situ hybridization was used to demonstrate that the mRNA for SC35 was localized in the same cells as the protein and, thus, to verify the immunohistochemical data. In implantation sites of rhesus monkeys, the cells that showed positive expression of the mRNA were the cells in the trophoblast shell, floating villus and decidua (Fig. 2n,o,p, respectively), the same cells that stained positively for the protein (Fig. $2 \mathrm{j}, \mathrm{k}, \mathrm{l}$, respectively). Control tissues in which sense probe replaced antisense showed no such staining (Fig. 2q).

\section{Western blot analysis of human endometrium}

As further verification of the immunostaining data, two samples of extracts of human endometrium during each of the proliferative (days 6 and 7) and secretory (days 19 and 

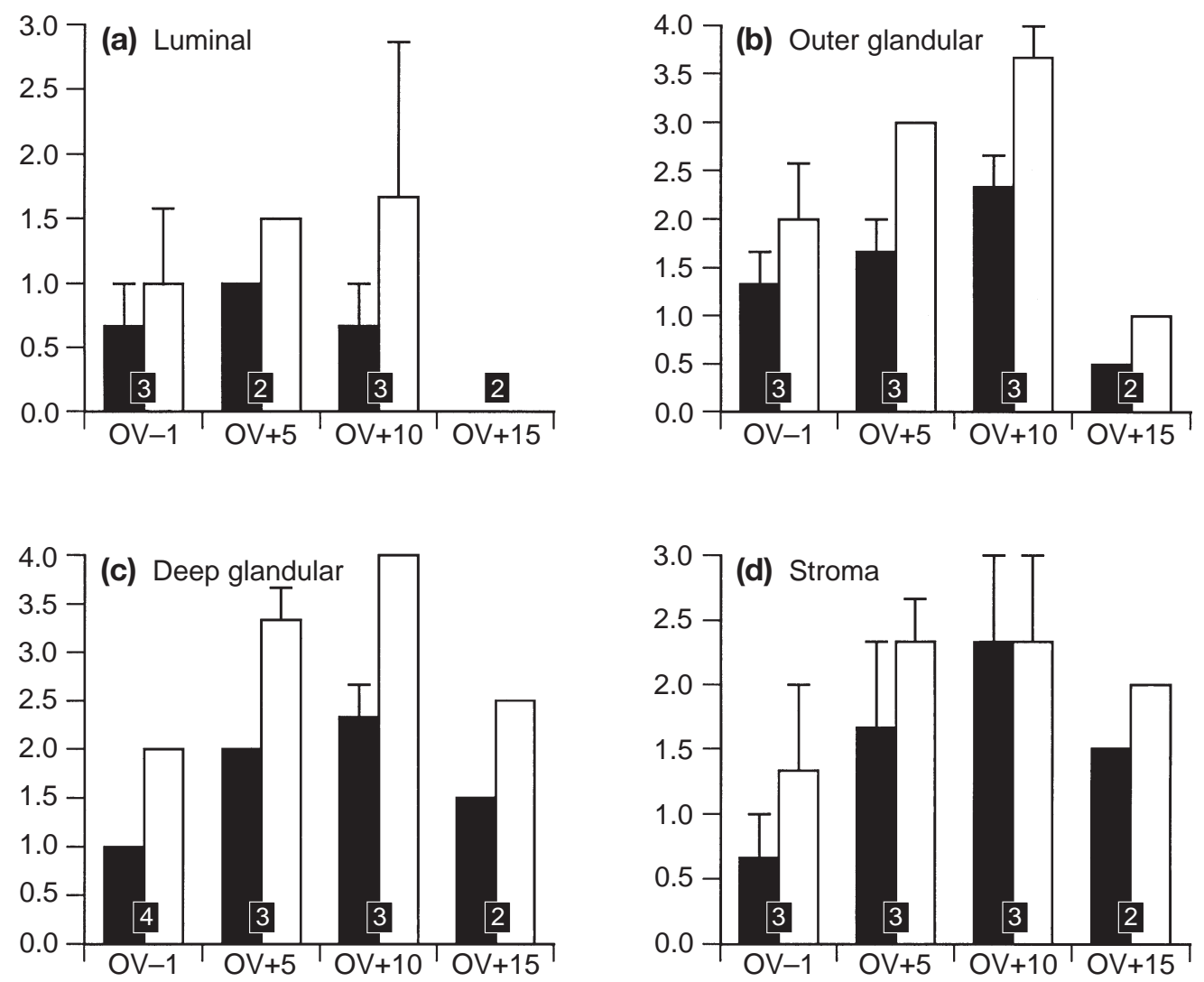

Fig. 3. Immunostaining of SC35 in rhesus monkey endometrium throughout the oestrous cycle. Mean intensity of immunostaining for SC35 ( $\mathbf{\square})$ and the proportion of stained cells within a cellular compartment $(\square)$ in monkey endometrium during the oestrous cycle. Ovulation (OV). OV $-1, \mathrm{OV}+5$, $\mathrm{OV}+10$ and $\mathrm{OV}+15$ represent the days immediately before $(-1)$ or 5, 10 and 15 days after ovulation. Cellular compartments are (a) luminal, (b) outer glandular and (c) deep glandular epithelium, respectively, and (d) stroma. Intensity of stain is represented on a scale of 0 (no stain) to 3 (maximum intensity). The proportion of cells stained within each cellular compartment is scored on a scale of 1-4 where $1=1-10 \%, 2=10-40 \%, 3=40-80 \%$ and $4=80-100 \%$ of the total cells. Data are expressed as mean \pm SEM. The number of animals included within each group is given within the histograms. Where no error bar is given and $n=3$, there is no variation between samples.

25) phases of the menstrual cycle were subjected to western blot analysis. In each case, a single band of protein was detected on the western blot at approximately $90 \mathrm{kDa}$ (Fig. 4). In accordance with the data (Fig. 1), substantially more SC35 protein was detected in the secretory phase than in the proliferative phase tissue.

\section{Discussion}

Previous studies have identified the splicing factor SC35 gene as one of a number of genes that is differentially expressed between implantation sites and inter-implantation sites in the mouse uterus and is present in much higher amounts at implantation sites between day 4.5 and day 8.5 (Nie et al., 2000c). In the present study, it was established that SC35 protein is present in the human and rhesus monkey endometrium, that the protein is increased during the secretory phase of the oestrous cycle compared with the

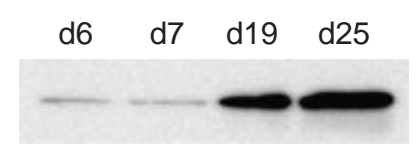

Fig. 4. Western blot analysis of SC35 in extracts from proliferative day 6 (d6) and day 7 (d7), and secretory day 19 (d19) and day 25 (d25) human endometrium. The band shown is approximately $90 \mathrm{kDa}$. No other bands were observed.

proliferative phase in both of these primates, and that it is present in the nucleus of both epithelial and stromal cells as well as pericytes associated with the vasculature. Interestingly, not only the intensity of immunoreactive protein, but also the proportion of cells in any one cellular compartment that stains for SC35, alters with the time of the oestrous cycle. This increase during the secretory phase was verified by western blot analysis and is in accordance with 
previous findings that progesterone is a positive regulator of SC35 gene expression in mouse endometrium (Nie et al., 2000c). The data indicate that there is a role for this splicing factor in preparation of a receptive uterus and in the provision of secreted proteins to support blastocyst development.

As it is not possible to examine early implantation sites in humans, such material was collected from pregnant rhesus monkeys and showed that there is a very precise expression pattern of SC35 during early placentation. In particular, both SC35 protein and mRNA are expressed strongly in cytotrophoblasts within the trophoblastic shell, in syncytiotrophoblast at the periphery of the cell column and in both cytotrophoblast and syncytiotrophoblast in the floating villi. In contrast, in the adjacent maternal decidua, only small amounts of SC35 immunoreactive protein were detected. Thus, it is postulated that at the site of trophoblast invasion, SC35 plays a role in alternative splicing of mRNAs to provide factors necessary for this invasion.

In the present study, the SC35 protein in human endometrium was detected by western blot analysis and had a molecular mass of approximately $90 \mathrm{kDa}$, indicating that in the endometrial extracts, the $35 \mathrm{kDa}$ protein remains bound to one or more components of the spliceosome. Importantly, SC35 protein is detected in tissue sections in discrete particles within the nuclei of cells, a feature previously shown to be characteristic of SC35 and not of other small nuclear ribonucleoprotein particles (snRNPs) (Fu and Maniatis, 1990). This feature verifies the specificity of the antibody used for SC35. Additional confirmation of the immunohistochemical data is provided by the detection of mRNA for SC35 in the same cells as the protein at implantation sites in the rhesus monkey.

The removal of introns from pre-mRNA precursors by splicing is a fundamental process that controls gene expression during differentiation and development, and is an essential and often regulated step in the expression of eukaryotic genes (Rio, 1992). It occurs in the nucleus in a large multi-component ribonucleoprotein complex, the spliceosome, which is composed of snRNPs and many nonsnRNP protein splicing factors (Kramer, 1996; Reed and Palandjian, 1997). Among the best-characterized nonsnRNP proteins are the SR family of splicing factors, which are highly conserved (Fu, 1995; Graveley, 2000). SR proteins are essential for constitutive splicing (Krainer et al., 1990). Although it was thought previously that there was considerable redundancy in function, it now appears that different SR proteins act on distinct exonic splicing enhancer elements (Schaal and Maniatis, 1999).

In addition to their roles in constitutive splicing, SR proteins are thought to be an important class of regulators for alternative splicing as they can modulate splice site selection in a concentration-dependent manner both in vitro and in transfected cells with artificial minigene constructs (Ge and Manley, 1990; Krainer et al., 1990; Fu et al., 1992; Zahler et al., 1992; Caceres et al., 1994; Cavaloc et al., 1994; Kanopka et al., 1996; Jiang et al., 1998). However, the notion that SR proteins may act as regulators for alternative splicing in cell physiology in vivo remains an unproven concept.

Recent functional studies have demonstrated important roles for some SR proteins, including SC35. Deletion of the SRp20 gene in the mouse blocked development at a very early stage such that preimplantation embryos failed to form blastocysts and died at the morula stage (Jumma et al., 1999), indicating that at least this SR protein may be required for cell viability. Conditional deletion of SC35 gene with the Cre-LoxP system in the thymus caused a defect in T-cell maturation (Wang et al., 2001) and also altered alternative splicing of CD45, a receptor tyrosine phosphatase known to be regulated by differential splicing during thymocyte development and activation. Thus, it is apparent that SC35 may function as a key regulator in Tcell-specific development pathways and that the function of CD45 is regulated by differential splicing rather than transcription during T-cell development and activation.

The other natural known target of the SC35-splicing activity is SC35 pre-RNA (Sureau et al., 2001); activation of splicing events leads to expression of unstable mRNA. In this context, in the mouse uterus, a number of alternatively spliced mRNA species of SC35 were identified (Nie et al., 2000c) and the expression of these was differentially regulated both during early pregnancy and by steroid hormones. Similar bands have been detected on northern blots for human SC35 splicing factor (Sureau and Perbal, 1994) and these have significantly different half-lifes. It is anticipated that in the primate uterus, as in that of the mouse, differential splicing of SC35 will occur during the differentiation and invasive events associated with implantation.

Among genes known to be important at implantation, many have spliced isoforms. These include cyclooxygenase-2 (Zuo et al., 1994), the prostaglandin receptors EP1 and EP3 (Pierce and Regan, 1998), the cell surface adhesion molecule CD44 (Behzad et al., 1994) and the oestrogen (Flouriot et al., 1998) and progesterone (Jeltsch et al., 1990) receptors. Thus, the action of SC35 may be critical in providing appropriate implantation-specific isoforms at this time.

The data presented here show expression of a critical splicing factor in the primate uterus. Regulation of this factor is finely tuned, both spatially and temporally, in the endometrium at the time when implantation would occur and in the trophoblast during placentation. These findings are in accordance with previous demonstration of its regulation at implantation sites in the mouse uterus and support a role for regulation of isoform formation by alternative splicing as an important mechanism necessary for embryo implantation. Disruption of such splicing could result in disorders of this process and could lead to infertility.

The authors are grateful to S. Panckridge for assistance with the figures and S. Park for assistance with preparation of the 
manuscript. The human endometrial tissue was generously provided by G. Kovacs, B. Vollenhoven and their patients and was collected by A. Clare and $\mathrm{H}$. Widjaja. This work was supported by the Rockefeller Foundation/World Health Organization Initiative on Implantation (A. L. Hampton and G-Q. Fu), the National Health and Medical Research Council of Australia (J. K. Findlay and L. A. Salamonsen), the Wellcome Trust (grant 52666), the CAS ChuangXin programme and '973' (G1999055901).

\section{References}

Behzad F, Seif MW, Campbell S and Aplin JD (1994) Expression of two isoforms of CD44 in human endometrium Biology of Reproduction $\mathbf{5 1}$ 739-747

Caceres JF, Stamm S, Helfman DM and Krainer AR (1994) Regulation of alternative splicing in vivo by overexpression of antagonistic splicing factor Science 265 1706-1709

Cavaloc Y, Popielarz M, Fuchs JP, Gattoni R and Stevenin J (1994) Characterization and cloning of the human splicing factor 9G8: a novel $35 \mathrm{kDa}$ factor of the serine/arginine protein family $E M B O$ Journal $\mathbf{1 3}$ 2639-2649

Dimitriadis E, Salamonsen LA and Robb L (2000) Expression of interleukin11 during the human menstrual cycle: coincidence with stromal cell decidualization and relationship to leukaemia inhibitory factor and prolactin Molecular Human Reproduction 6 907-914

Flouriot G, Griffin C, Kenealy M, Sonntag-Buck V and Gannon F (1998) Differentially expressed mRNA isoforms of the human estrogen receptor-alpha gene are generated by alternative splicing and promoter usage Molecular Endocrinology 12 1939-1954

Fu X-D (1995) The superfamily of arginine/serine-rich splicing factors $R N A$ $1663-680$

Fu X-D and Maniatis T (1990) Factor required for mammalian spliceosome assembly is localized to discrete regions in the nucleus Nature $\mathbf{3 4 3}$ 437-441

Fu X-D and Maniatis T (1992) The 35 kDa mammalian splicing factor SC35 mediates specific interactions between $\mathrm{U} 1$ and $\mathrm{U} 2$ small nuclear ribonucleoprotein particles at the $3^{\prime}$ splice site Proceedings National Academy of Sciences USA 89 1725-1729

Fu X-D, Mayeda A, Maniatis T and Krainer AR (1992) General splicing factors SF2 and SC35 have equivalent activities in vitro, and both affect alternative $5^{\prime}$ and $3^{\prime}$ 'splice site selection Proceedings National Academy of Sciences USA 8911 224-11 228

Ge H and Manley JL (1990) A protein factor, ASF, controls cell-specific alternative splicing of SV40 early pre-mRNA in vitro. Cell 62 25-34

Graveley BR (2000) Sorting out the complexity of SR protein functions $R N A$ 6 1197-1211

Hambartsoumian E (1998) Endometrial leukemia inhibitory factor (LIF) as a possible cause of unexplained infertility and multiple failures of implantation Journal of Reproduction and Fertility 39 137-143

Jeltsch JM, Turcotte B, Garnier JM, Lerouge T, Krozowski Z, Gronemeyer H and Chambon P (1990) Characterization of multiple mRNAs originating from the chicken progesterone receptor gene. Evidence for a specific transcript encoding form A Journal of Biological Chemistry 265 3967-3974

Jiang ZH, Zhang WJ, Rao Y and Wu JY (1998) Regulation of Ich-1 premRNA alternative splicing and apoptosis by mammalian splicing factors Proceedings National Academy of Sciences USA 95 9155-9160

Jumma H, Wei G and Nielsen PJ (1999) Blastocyst formation is blocked in mouse embryos lacking the splicing factor SRp20 Current Biology $\mathbf{9}$ 899-902

Kanopka A, Muhlemann O and Akusjarvi G (1996) Inhibition by SR proteins of splicing of a regulated adenovirus pre-mRNA Nature 381 535-538
Krainer AR, Conway GC and Kozak D (1990) The essential pre-mRNA splicing factor SF2 influences $5^{\prime}$ splice site selection by activating proximal sites Cell 62 35-42

Kramer A (1996) The structure and function of proteins involved in mammalian pre-mRNA splicing Annual Reviews of Biochemistry 65 $367-409$

Kumar S, Zhu LJ, Polihronis M et al. (1998) Progesterone induces calcitonin gene expression in human endometrium within the putative window of implantation Journal of Clinical Endocrinology and Metabolism 83 4443-4450

Liang P and Pardee AB (1992) Differential display of eukaryotic mRNA by means of the polymerase chain reaction Science 257 967-971

Liang P and Pardee AB (1993) Distribution and cloning of eukaryotic mRNAs by means of differential display: refinement and optimization Nucleic Acids Research 14 3269-3275

Nie G-Y, Li Y, Wang J, Minoura H, Findlay JK and Salamonsen LA (2000a) Complex regulation of calcium-binding protein D9k (Calbindin-D9k) in the mouse uterus during early pregnancy and at the site of embryo implantation Biology of Reproduction 62 27-36

Nie G-Y, Li Y, Hampton AL, Salamonsen LA, Clements JA and Findlay JK (2000b) Identification of monoclonal non-specific suppressor factor beta (MNSFbeta) as one of the genes differentially expressed at implantation sites compared to inter-implantation sites in the mouse uterus Molecular Reproduction and Development 55 351-363

Nie G-Y, Li Y, Batten L, Griffiths B, Wang J, Findlay JK and Salamonsen LA (2000c) Uterine expression of alternatively spliced mRNAs of mouse splicing factor SC35 during early pregnancy Molecular Human Reproduction 6 1131-1139

Pierce KL and Regan JW (1998) Prostanoid receptor heterogeneity through alternative mRNA splicing Life Sciences 62 1479-1483

Reed R and Palandjian L (1997) Spliceosme assembly. In Eurkaryotic mRNA Processing pp 103-129 Ed. AR Krainer. Oxford University Press, Oxford

Rio DC (1992) RNA processing Current Opinions in Cell Biology 4 444-452

Schaal TD and Maniatis T (1999) Multiple distinct splicing enhancers in the protein-coding sequences of a constitutively spliced pre-mRNA Molecular and Cellular Biology 19 261-273

Sureau A and Perbal B (1994) Several mRNAs with variable 3' untranslated regions and different stability encode the human PR264/SC35 splicing factor Proceedings National Academy of Sciences USA 91 932-936

Sureau A, Gattoni R, Dooghe Y, Stevenin J and Soret J (2001) SC35 autoregulates its expression by promoting splicing events that destabilize its mRNAs EMBO Journal 20 1785-1796

Vogiagis D and Salamonsen LA (1999) The role of leukemia inhibitory factor (LIF) in the establishment of pregnancy Journal of Endocrinology $160181-190$

Wang HY, Xu X, Ding JH, Bermingham JR, Jr and Fu XD (2001) SC35 plays a role in T cell development and alternative splicing of CD45 Molecular Cell 7 331-342

Zahler AM, Lane WS, Stolk JA and Roth MB (1992) SR proteins: a conserved family of pre-mRNA splicing factors Genes and Development 6 837-847

Zuo J, Lei ZM, Rao CV, Pietrantoni M and Cook VD (1994) Differential cyclooxygenase- 1 and -2 gene expression in human myometria from preterm and term deliveries Journal of Clinical Endocrinology and Metabolism 79 894-899

Received 19 December 2001.

First decision 6 February 2002.

Revised manuscript received 10 April 2002.

Accepted 16 April 2002. 